\title{
Editorial
}

\section{Sensing and Intelligent Perception in Robotic Applications}

\author{
Changhai Ru, ${ }^{1}$ Xuping Zhang, ${ }^{2}$ Yajing Shen, ${ }^{3}$ and Yong Zhang ${ }^{4}$ \\ ${ }^{1}$ Research Center of Robotics and Micro System, Soochow University, Soochow, Jiangsu 215000, China \\ ${ }^{2}$ School of Engineering-Structural Mechanics, Aarhus University, 8000 Aarhus C, Denmark \\ ${ }^{3}$ Department of Mechanical and Biomedical Engineering, City University of Hong Kong, 83 Tat Chee Avenue, \\ Kowloon Tong, Hong Kong \\ ${ }^{4}$ School of Aerospace Engineering, Georgia Institute of Technology, Atlanta, GA 30332, USA \\ Correspondence should be addressed to Changhai Ru; rchhai@gmail.com
}

Received 29 July 2015; Accepted 30 July 2015

Copyright (C) 2016 Changhai Ru et al. This is an open access article distributed under the Creative Commons Attribution License, which permits unrestricted use, distribution, and reproduction in any medium, provided the original work is properly cited.

This special issue began in June 2014 and its submission system was closed in December 2014. The authors really appreciate the efforts of those reviewers in finalizing the review process of all the received submissions in our special issue. These accepted papers covered our special issue's several study aspects such as sensors calibration method, sensors information integration and fusion, vision sensor and vision algorithm in motive object tracking, and integrated navigation technology. For example, about vision sensors, some paper showed how a novel algorithm based robot vision system would be applied in an objects sorting process. Some paper showed how a Kinect based vision system would be used in a mine rescue robot in a low illuminous environment. Some papers discussed some novel vision method for the indoor robot path planning. About force sensors, some paper proposed a force-reflected nonlinear multilateral teleoperation system and discussed how to solve the control stability and time-varying delays. About information fusion and estimation method, some paper proposed Bayesian prediction of sequential probability matrices method to detect pedestrian in crowded environments. Some paper proposed a phasepath volume Kalman filtering method for target centroid position estimation. And some paper proposed a method to evaluate novel radar based scanning method. We tried our best to show the latest research results in the research fields above in our special issue. And we hope this special issue could be of some help to every reader. Thank you all for your attention.

Changhai Ru

Xuping Zhang

Yajing Shen

Yong Zhang 


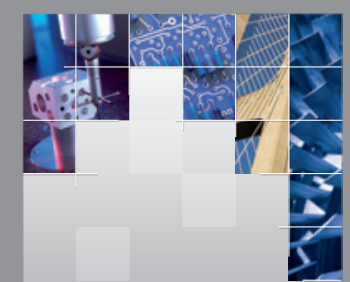

\section{Enfincering}
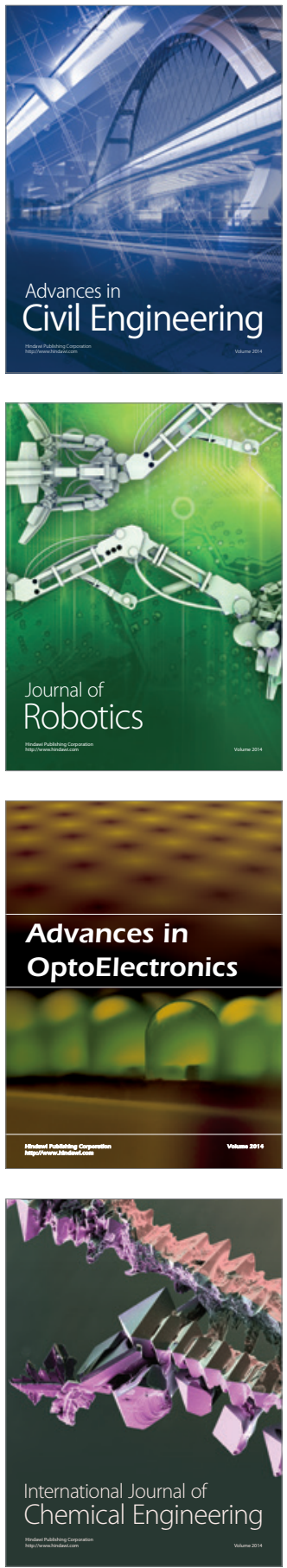

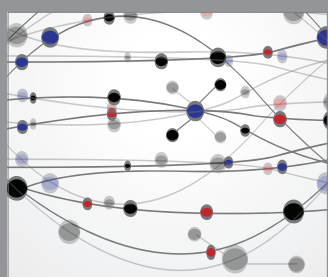

The Scientific World Journal

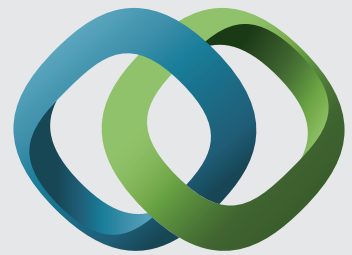

\section{Hindawi}

Submit your manuscripts at

http://www.hindawi.com
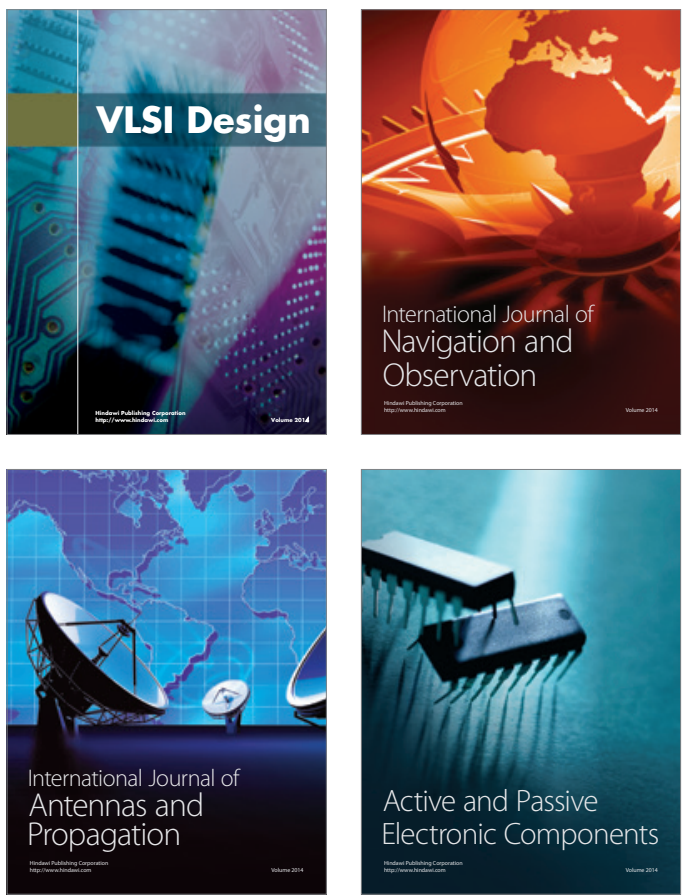
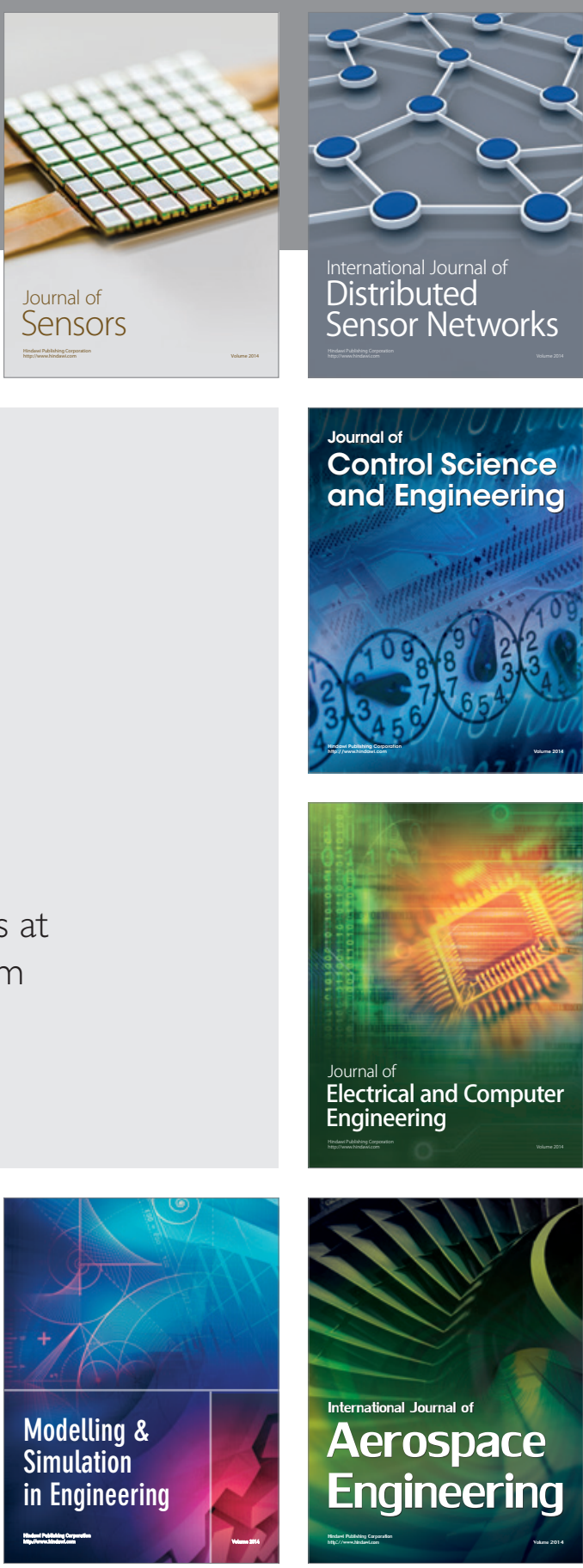

International Journal of

Distributed

Sensor Networks

Journal of

Control Science

and Engineering
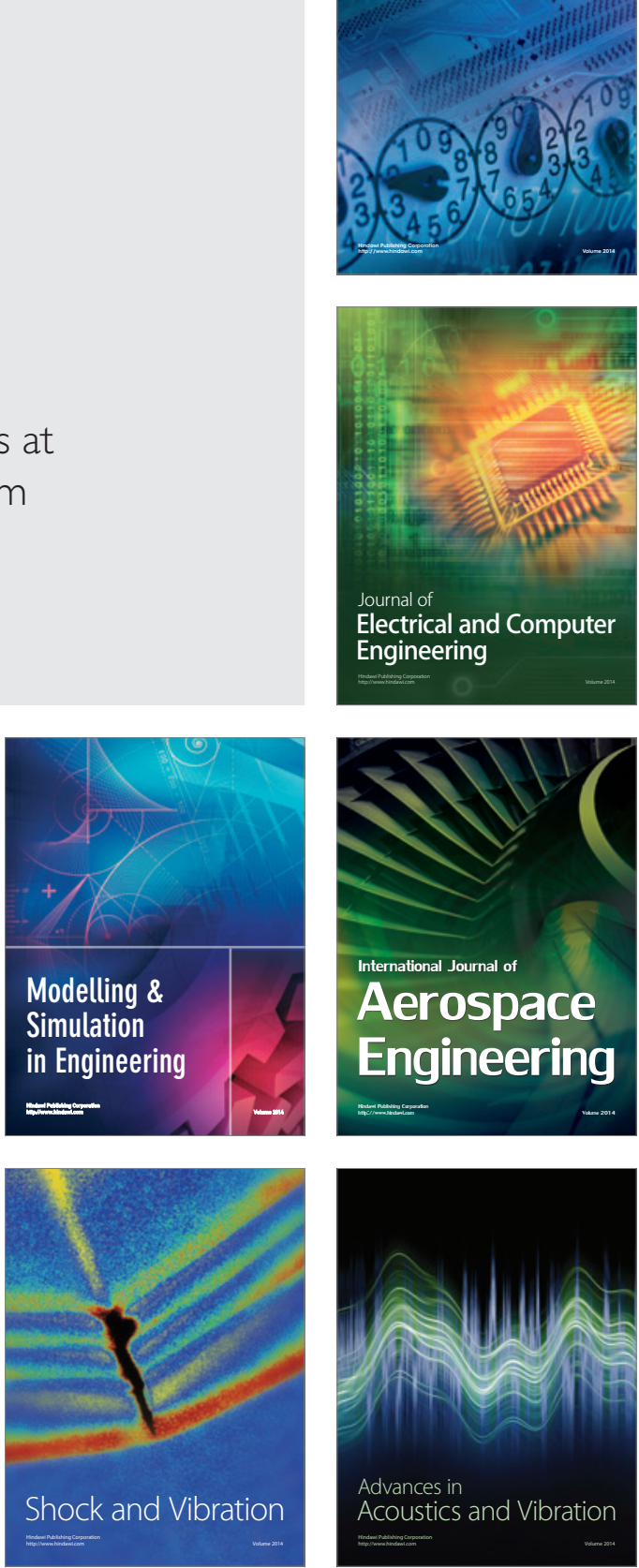